\title{
COMUNICACIÓN
}

\section{Piojos (Phthiraptera: Insecta) de aves de la familia Laridae (Aves: Charadriiformes) en Chile}

\author{
DANIEL GONZÁLEZ-ACUÑA*, CHRISTOF FISCHER*, RICARDO PALMA**, LUCILA MORENO*, \\ CARLOS BARRIENTOS*, LISANDRO MUÑOZ*, KAREN ARDILES* y ARMANDO CICCHINO***'
}

\section{LICE (PHTHIRAPTERA: HEXAPODA) FROM MARINE BIRD BELONGING TO THE FAMILY LARIDAE FROM CHILE}

Six species of lice (Phthiraptera: Philopteridae, Menoponidae) were collected from four marine bird species belonging to the family Laridae from the Chilean coast. Saemundssonia sternae (Linnaeus, 1758) and Quadraceps sellatus (Burmeister,1838) collected from the boreal sea gull (Sterna hirundo, Linnaeus, 1758), Saemundssonia lari (O, fabricius, 1780), Quadraceps punctatus (Burmeister, 1838) and Q. ornatus (Grube, 1851) found on the Dominican sea gull (Larus dominicanus, Lichtenstein, 1832); Q, punctatus collected from garuma sea gull (L. modestus, Tschudi, 1843) and S. lari and Actornithophilus piceus lari (Packard,1870) found on Franklin sea gull (L. pipixcan, Wagler,1831). All these species of Phthiraptera are first reports in Chile.

Key words: Phthiraptera, Marine birds parasites, Sea gull, Lice, Chile.

\section{INTRODUCCIÓN}

Los piojos (Insecta: Arthropoda: Phthiraptera) son parásitos de aves y mamíferos que comparten una estrecha relación con sus hospedadores ${ }^{1}$, lo cual ha servido para realizar estudios de coevolución $^{2}$, de la comunidad ecológica ${ }^{3}$, y de poblaciones genéticas ${ }^{4,5}$.

Además, contribuyen a elucidar relaciones filogenéticas entre miembros de la clase Aves, debido a que muchos géneros de piojos están restringidos a ciertos órdenes de aves, y a su vez, algunas especies dentro de determinados géneros pueden estar restringidas a una sola especie de hospedador o a varias subespecies ${ }^{6,7}$. A través de los piojos se puedencomprender las conductas de los hospedadores, lo cual debería considerarse en iniciativas de biodiversidad y conservación ${ }^{8}$. Esto es de gran interés en Chile, ya que la información referente a nuestra biodiversidad es limitada 9 , y en el caso de los Phthiraptera los estudios han sido reducidos y esporádicos, siendo la mayoría realizados por investigadores extranjeros.

En Chile, los estudios de piojos de aves de la familia Laridae se han restringido al reporte de la especie Haffneria grandis (Piaget, 1880) (como Perineus grandis) sobre Stercorarius $\mathrm{sp}^{10}$, y a la descripción de Heteromenopon macrurum (Eichler, 1952) sobre Sterna paradisaea Pontoppidan (= Sterna macrura Naumann), aunque este registro se debió a una contaminación de piojos provenientes de una especie de loro (Orden Psittaciformes) $^{11,12}$.

\footnotetext{
* Facultad de Medicina Veterinaria, Universidad de Concepción, Casilla 537, Chillán, Chile. danigonz@udec.cl

** Museum of New Zealand Te Papa Tongerawa, P.O. Box 467, Wellington, New Zealand.

** Universidad de Mar del Plata, Castelli 4117, 7600 Mar del Plata, Argentina.
} 


\section{MATERIAL Y MÉTODO}

Con el objetivo de determinar las especies de piojos presentes en aves de la Familia Laridae en Chile, desde agosto del año 2002 hasta diciembre del 2005 se han aislado piojos de aves lesionadas que llegan al centro de rescate de la Facultad de Medicina Veterinaria de la Universidad de Concepción ( $\mathrm{n}=5)$, aves capturadas dentro del marco del proyecto SAG-APA («Proyecto de Nacional de Vigilancia en enfermedades aviares exóticas de la lista A de la Organización Mundial de Sanidad Animal y Bronquitis Infecciosa») (n =6) y muestras colectadas de aves preservadas en el Museo Nacional de Historia Natural de Santiago de Chile $(\mathrm{n}=18)$ (Tabla 1).

Los piojos fueron aislados y almacenados en alcohol al 70\%. Posteriormente, fueron montados en Bálsamo de Canadá ${ }^{13}$. Para la identificación de cada ejemplar se usaron claves taxonómicas y descripciones dadas por distintos autore ${ }^{13-20}$. El material estudiado fue depositado en la colección zoológica de la Facultad de Medicina Veterinaria, Universidad de Concepción.

\section{RESULTADOS Y DISCUSIÓN}

Seis especies de Phthiraptera fueron aisladas de cuatro especies de aves (Charadriiformes: Laridae) examinadas. Las medidas de los ejemplares mejor conservados se presentan en la Tabla 2.
Sobre el gaviotín boreal (Sterna hirundo Linnaeus, 1758) se aislaron siete piojos, cinco correspondientes a Saemundssonia sternae (Linnaeus, 1758) y dos a Quadraceps sellatus (Burmeister, 1838) (Tabla 1). La especie $S$. sternae ya ha sido registrada sobre cuatro especies de gaviotines ${ }^{13}$, incluyendo $S$. hirundo.

El género Quadraceps (Clay \& Meinertzhagen, 1939) contiene una gran cantidad de especies y subespecies parásitas sobre miembros del orden Charadriiformes (chorlos, gaviotines y gaviotas $)^{13}$. En Chile, no existen registros de especies del género Quadraceps, por lo tanto las dos especies reportadas en este trabajo representan los primeros registros para el país. Sobre Larus dominicanus (Lichtenstein, 1832) se obtuvieron tres especies de Phthiraptera: un ejemplar de Quadraceps punctatus (Burmeister, 1838) uno de Quadraceps ornatus (Grube, 1851) y 12 de Saemundssonia lari (O. Fabricius, 1780). Ambas especies de Quadraceps, $Q$. punctatus y $Q$. ornatus se han registrado frecuentemente sobre muchas especies de la familia Laridae, incluyendo L. dominicanus, y han sido subdivididas en varias subespecies ${ }^{13}$. El presente reporte constituye el primer registro de $Q$. punctatus y de $Q$. ornatus sobre $L$. dominicanus para Chile, como así también lo es el registro de Q. punctatus sobre Larus modestus (Tschudi, 1843).

Saemundssonia lari es una especie morfológicamente variable que parasita a 36 aves

Tabla 1. Piojos aislados de aves de la familia Laride según hospedador, cantidad, sexo y origen de la colecta

\begin{tabular}{|c|c|c|c|c|c|c|}
\hline \multirow[t]{2}{*}{$\begin{array}{l}\text { Especie de } \\
\text { hospedador }\end{array}$} & \multirow[t]{2}{*}{$\begin{array}{c}\text { Procedencia } \\
\text { del hospedador }\end{array}$} & \multirow[t]{2}{*}{$\begin{array}{c}\text { Especie/subespecie } \\
\text { de piojo }\end{array}$} & \multicolumn{3}{|c|}{$\begin{array}{c}\mathrm{N}^{\circ} \text { de ejemplares } \\
\text { analizados por sexo }\end{array}$} & \multirow[t]{2}{*}{ Total } \\
\hline & & & Macho & Hembra & Ninfa & \\
\hline \multirow[t]{2}{*}{ Sterna hirundo } & Santo Domingo & Saemundsonia sternae & 3 & 1 & 1 & 5 \\
\hline & & Quadraceps sellatus & 1 & 1 & & 2 \\
\hline \multirow{3}{*}{ Larus dominicanus } & Concepción & Saemundsonia lari & 8 & 4 & & 12 \\
\hline & Isla Roberto & Quadraceps punctatus* & 1 & & & 1 \\
\hline & Valparaíso & Quadraceps ornatus* & & 1 & & 1 \\
\hline Larus modestus & San Antonio & Quadraceps punctatus* & 1 & & & 1 \\
\hline \multirow{4}{*}{ Larus pipixcan } & Santo Domingo & Saemundsonia lari & 2 & & & 2 \\
\hline & Río Lluta & & 1 & & & 1 \\
\hline & Río Maipo & & 1 & & & 1 \\
\hline & Santo Domingo & Actornithophilus piceus lari & & 1 & & 1 \\
\hline Total & & & 18 & 8 & 1 & 27 \\
\hline
\end{tabular}

* Material colectado de aves preservadas en el Museo Nacional de Historia Natural de Chile. 
Piojos de aves de la familia Laridae (Aves: Charadriiformes) en Chile - D. González et al.

Tabla 2. Medidas corporales promedio en micrómetros $(\mu \mathrm{m})$ de las especies de piojos aisladas de aves de la familia Laridae

\begin{tabular}{|c|c|c|c|c|c|c|c|c|}
\hline Especie/subespecie & Sexo $n$ & $\begin{array}{l}\text { Largo } \\
\text { cabeza }\end{array}$ & $\begin{array}{l}\text { Ancho } \\
\text { cabeza }\end{array}$ & $\begin{array}{c}\text { Largo } \\
\text { tórax }\end{array}$ & $\begin{array}{l}\text { Ancho } \\
\text { tórax }\end{array}$ & $\begin{array}{c}\text { Largo } \\
\text { abdomen }\end{array}$ & $\begin{array}{c}\text { Ancho } \\
\text { abdomen }\end{array}$ & $\begin{array}{l}\text { Largo } \\
\text { total } \\
\text { cuerpo }\end{array}$ \\
\hline \multicolumn{9}{|l|}{ Saemundssonia } \\
\hline sternae & M 3 & 567,65 & 552,90 & 380,86 & 371,76 & 729,55 & 621,59 & 1678,06 \\
\hline & $\mathrm{H}$ & & & & 76 & 00 & 34 & 1885,77 \\
\hline \multicolumn{9}{|l|}{ Quadraceps } \\
\hline sellatus & H 2 & 485,06 & 372,12 & 424,59 & 318,56 & 1218,12 & 453,48 & 2127,77 \\
\hline \multicolumn{9}{|l|}{ Quadraceps punctatus } \\
\hline $\begin{array}{l}\text { (ex Larus dominicanus) } \\
\text { Saemund sonia lari }\end{array}$ & M 1 & 533,98 & 431,78 & 500,99 & 363,42 & - & 600,39 & - \\
\hline Saemundssonia lari & H 4 & & & & & & & \\
\hline \multirow{2}{*}{ (ex Larus dominicanus) } & M 8 & 564,75 & 562,51 & 370,89 & 384,42 & 858,33 & 630,39 & - \\
\hline & H 4 & 602,88 & 622,30 & 451,83 & 427,76 & 1021,07 & 755,71 & 2075,79 \\
\hline \multirow{3}{*}{$\begin{array}{l}\text { Quadraceps ornatus } \\
\text { Saemundssonia lari }\end{array}$} & H 1 & 915,25 & 797,38 & 416,02 & 767,00 & 2010,78 & 621,48 & 3342,05 \\
\hline & M 1 & 610 & 591,29 & 498,43 & 41 & 92. & 738,56 & 2032,60 \\
\hline & H 2 & 607,09 & 664,88 & 468,4 & 453,28 & 1165,48 & 970,77 & 2240,97 \\
\hline $\begin{array}{l}\text { Quadraceps punctatus fe } \\
\text { (ex Larus modestu) }\end{array}$ & M 1 & 649,49 & 485,22 & 763,77 & 509,40 & 2183,24 & 602,11 & 3596,50 \\
\hline
\end{tabular}

$\mathrm{M}=$ macho, $\mathrm{H}=$ hembra

de la familia Laridae ${ }^{13}$, incluyendo L. dominicanus. El presente trabajo reporta la especie $S$. lari por primera vez en Chile, aislada de L. dominicanus y de L. pipixcan (Wagler, 1831).

Finalmente, sobre la gaviota de Franklin $(L$. pipixcan), se registra la subespecie Actornithophilus piceus lari (Packard, 1870). Esta subespecie ha sido mencionada para L. pipixcan en Argentina ${ }^{21}$ y en América del Norte ${ }^{22}$. El presente registro es la primera cita del género Actornithophilus (Ferris, 1916) para Chile.

La cuarta especie de piojo comúnmente registrada sobre miembros de la familia Laridae es Austromenopon transversum (Denny, 1842), pero no fue hallada durante el presente estudio.

\section{RESUMEN}

Seis especies de piojos (Phthiraptera: Philopteridae, Menoponidae) fueron colectadas sobre cuatro especies de aves marinas de la familia Laridae en la costa de Chile. Se registran: Saemundssonia sternae (Linnaeus, 1758) y Quadraceps sellatus (Burmeister, 1838) sobre el gaviotín boreal (Sterna hirundo, Linnaeus, 1758); Saemundssonia lari (O. Fabricius, 1780), Quadraceps punctatus (Burmeister, 1838) у Quadraceps ornatus (Grube, 1851) parasitando a la gaviota dominicana (Larus dominicanus Lichtenstein, 1832); Q. punctatus sobre la gaviota garuma (L. modestus Tschudi, 1843); S. lari y Actornithophilus piceus lari (Packard, 1870) sobre la gaviota de Franklin (L. pipixcan Wagler, 1831). Todas estas especies de Phthiraptera constituyen nuevos registros para Chile.

\section{REFERENCIAS}

1.- MARSHALL A G. The ecology of ectoparasitic insects. Academic Press. New YorK, USA. 1981.

2.- HAFNER M S, PHILIP D S, VILLABLANCA F X, et al. Disparate rates of molecular evolution in cospeciating hosts and parasites. Science 1994; 265 : 1087-90.

3.- CLAYTON D H, DROWN D M. Critical evaluation of five methods for quantifying chewing lice (Insecta: Phthiraptera). J Parasitol 2001; 87: 1291-300.

4.- JOHNSON K P, ADAMS R J, CLAYTON D H. Molecular systematics of Goniodidae (Insecta: Phthiraptera). J Parasitol 2001; 87: 862-9.

5.- JOHNSON K P, WILLIAMS B L, DROWN D M. The population genetics of host specificity: genetic differentiation in dove lice. Mol Ecol 2002; 11: 25-38.

6.- CLAYTON D H. Mate choice in experimentally parasitized rock doves: Lousy Males Lose. Amer Zool 1990; 30: 251-62.

7.- PAGE R D M, CRUICKSHANK R, DICKENS M, et al. Phylogeny of "Philoceanus complex" seabird lice (Phthiraptera: Ischnocera) inferred from mitochondrial DNA sequences. Mol Phyl Evol 2004; 30: 633-52. 
8.- HAHN D C, PRICE R D, OSENTON P C. Use of lice to identify cowbird hosts. Auk 2000; 117: 943-51.

9.- SIMONETTI J A, ARROYO M T K, SPOTORNO A E, LOZADA E. Diversidad biológica de Chile. Comité Nacional de Diversidad Biológica, Comisión Nacional de Investigación Científica y Tecnológica. Santiago, Chile. 1995.

10.- CARRIKER M (Jr). Descriptions of new and little known species of Mallophaga (Insecta) from maritime hosts of Chile, South America. pp. 6-10. Publicaciones del Centro de Estudios Entomológicos. $\mathrm{N}^{\circ} 6$. Universidad Católica de Valparaíso. Valparaíso, Chile. 1964.

11.- PRICE R D, BEER J R. The genus Heteromenopon (Mallophaga: Menoponidae), with description of a new subgenus and six new species. Ann Entomoll Soc Amer 1967; 60: 328-38.

12.- PALMA R L. Acerca del verdadero hospedador de Heteromenopon (Heteromenopon) macrurum (Eichler, 1952) (Mallophaga, Menoponidae). Physis (Sección C) $1975 ; 34: 113-5$.

13.- PRICE R D, HELLENTHAL R A, PALMA R L. World checklist of chewing lice with host associations and keys to families and genera. pp. 1-448. In: The chewing lice: world checklist and biological overview. Illinois Natural History Survey Special Publication.USA. 2003.

14.- CLAY T. Species of the genus Saemundssonia (Mallophaga) from the Sterninae. Am Mus Novit 1949; (1409): 1-25.

15.- CLAY T. Key to the species of Austromenopon Bedford (Mallophaga) parasitic on the Charadriiformes. Proc. R. Entomol. Soc. Lond. (Series B) 1959; 28: 157-68.

16.- CLAY T. A key to the species of Actornithophilus Ferris with notes and descriptions of new species. Bull Br Mus (Natural History) Entomol 1962; 11: 189-244, pls 4-11.

17.- WARD R A. Biting lice of the genus Saemundssonia (Mallophaga: Philopteridae) occuring on terns. Proc U S Natl Mus 1955; 105: 83-100.

18.- EMERSON K C, PRICE R. D. The identity of Quadraceps crassipedalis (Harrison) and two new species of Quadraceps (Mallophaga: Philopteridae). Proc Entomol Soc Wash 1985; 87: 395-401.

19.- PALMA R L. A New Synonymy and New Records of Quadraceps (Insecta: Phthiraptera: Philopteridae) from the Galapagos Islands. New Z J Zool 1995; 22: 217 22.

20.- CICCHINO A, GONZÁLEZ D, FIGUEROA R. En: Curso-Taller. Estado del conocimiento de los Phthiraptera (Hexapoda: Insecta) ectoparásitos de aves y mamíferos en el cono sur de Sudamérica. Su importancia científica, veterinaria, médica, económica y estética. 17-18-19 de enero 2005. Universidad de Concepción. Fac Med Vet Chillán, Chile. 2005.

21.- CICCHINO A C, CASTRO D DEL C. Amblycera. pp: 34-103 e Ichnocera pp: 104-137 En: J. J. Morrone y S. Coscarón (Ed.) Biodiversidad de artrópodos Argentinos. Ediciones Sur. La Plata, Argentina. 1998.

22.- EMERSON K C. Checklist of the Mallophaga of North America (North of Mexico) Part II. Suborder Amblycera. Dugway (Utah): Deseret Test Center, 1972; $118 \mathrm{pp}$.

Agradecimientos: A Julissa Heria y Charif Tala, quienes colectaron gentilmente Phthiraptera de aves capturadas durante el desarrollo del Proyecto SAG-APA. A Juan Carlos Torres Mura por permitir revisar el material ornitológico del Museo Nacional de Historia Natural de Santiago de Chile. 